\title{
Germanica
}

\section{Bible et mythe dans les premières œuvres de Louis Couperus}

De bijbel en het mythische element in de vroege werken van Louis Couperus

\section{Marjan Krafft-Groot}

\section{OpenEdition}

\section{Journals}

Édition électronique

URL : http://journals.openedition.org/germanica/1942

DOI : 10.4000/germanica. 1942

ISSN : 2107-0784

Éditeur

Université de Lille

\section{Édition imprimée}

Date de publication : 31 décembre 1996

Pagination : 49-66

ISSN : 0984-2632

\section{Référence électronique}

Marjan Krafft-Groot, "Bible et mythe dans les premières œuvres de Louis Couperus », Germanica [En ligne], 19 | 1996, mis en ligne le 04 juin 2013, consulté le 06 octobre 2020. URL : http://

journals.openedition.org/germanica/1942 ; DOI : https://doi.org/10.4000/germanica.1942

Ce document a été généré automatiquement le 6 octobre 2020.

(C) Tous droits réservés 


\title{
Bible et mythe dans les premières œuvres de Louis Couperus
}

\author{
De bijbel en het mythische element in de vroege werken van Louis Couperus
}

\author{
Marjan Krafft-Groot
}

1 Louis Couperus (1863-1923) s'est fait une solide réputation littéraire par son roman naturaliste Eline Vere (1889). C'est une autre œuvre, cependant, qui est traduite dès 1891 à Londres et dès 1895 à Prague ${ }^{1}$ - bientôt suivie par la Pologne : Noodlot (Ossud) (1890). Le décalage entre ces œuvres met en évidence autant l'évolution dans les goûts littéraires et les critères distinctifs que l'intérêt des premiers romans, où valeurs littéraires, sources bibliques et éléments autobiographiques se mêlent, avant de révéler l'auteur et son style.

2 En étudiant l'œuvre de Couperus, on est frappé par l'étrange sensation de menace qui se manifeste sous différentes formes. Une force occulte hante et détruit dans De Stille Kracht ( $L a$ Force occulte) écrit en 1900 la vie de la famille d'un résident général hollandais en Indonésie. Elle sera présente ailleurs, comme dans De Berg van Licht (La Montagne de lumière) (1906) où Héliogabale, le jeune enfant-empereur androgyne est hanté par une menace qui, depuis sa naissance, détermine le cours de sa vie. Ce sentiment domine, pour ainsi dire, tous les héros coupériens : ce qu'en 1888-1889 Eline Vere, jeune femme de la haute bourgeoisie de la Haye, ressent comme une fatalité dans le roman qui porte son nom, se présente comme une force silencieuse dans De Stille Kracht.

3 Guus Houtzager compare, en 1992 dans De ongrijpbare Kracht van het Noodlot (La Force invisible de la fatalité) $)^{2}$, cette force silencieuse à un pouvoir occulte qui entraîne la fatalité, présage de la chute. Elle est là où le mal a pris racine. C'est l'aboutissement du mal qui se manifeste par le malheur et les catastrophes. Elle s'abat sur une collectivité, grande ou petite, comme une force surnaturelle inévitable. Cette force est pour Houtzager la punition infligée par un pouvoir supérieur. Elle est essentielle dans l'œuvre de Couperus et se dégage, dans les années 1890, d'une symphonie de récits où les éléments bibliques s'harmonisent sur un arrière-plan qui utilise l'Ancien Testament comme instrument de percussion. 
Eline Vere est victime de la fatalité. Le roman se situe dans le contexte fin-de-siècle de l'aristocratie de La Haye où l'héroïne est vouée, de par ses ancêtres et son milieu social, à sombrer dans le malheur. Pourtant, le roman n'est pas exclusivement déterministe et naturaliste ${ }^{3}$. Il en possède, certes, les particularités, mais, à la différence de Zola, il présente aussi le moyen d'échapper à la fatalité. Houtzager mentionne l'importance du rôle de St. Clare, riche américain qui, soutenant que la fatalité n'existe pas, donne à Eline l'ultime chance d'échapper par le mariage au cercle vicieux de ce qui représente pour elle la fatalité. Cette idée de fatalité lui avait d'ailleurs été soufflée par Vincent Vere, son cousin germain dont elle tombera amoureuse. Force est de constater que ce personnage de St. Clare dont le nom évoque le nouveau monde, voit clair/Clare - en elle comme dans le monde - et représente la réalité non romancée. Le préfixe Saint ajoute au nom la connotation de ce qui est bon et juste et qui pourrait remettre Eline dans le droit chemin autant que sur la bonne voie. Ses paroles s'inspirent de la philosophie théosophique largement répandue aux Pays-Bas par Annie Besant ${ }^{4}$.

Cette Annie Besant répand l'idée selon laquelle chaque individu tient entre les mains son propre destin. Cette idée se révèle importante dans la structuration des récits : elle nous guide vers la réponse à la problématique coupérienne des années 1890 . Pendant cette période, en effet, le héros va progressivement se faire et devenir une structure symbolique, avec des variantes selon les œuvres. L'œuvre de jeunesse de Couperus reflète ainsi l'évolution du personnage vers un accomplissement positif, synonyme de perfection. St. Clare et le mouvement théosophique venus des États-Unis s'inscrivent dans un courant rédempteur, préparant le mysticisme fin-de-siècle dont l'auteur parle dans Metamorfose (1897). Cela ne veut pas dire que Couperus ait adhéré à la théosophie : dans la même œuvre encore, considérée comme auto-biographique, Hugo Aylva, le héros en quête de bonheur, reconnaît s'être inspiré de cette philosophie jusqu'à un certain point seulement, pour en utiliser des éléments dans le livre qu'il rédige sous le titre de Metamorfose, livre lui aussi autobiographique et profession de foi :

Et il crut, avec la religion. Ce n'était pas uniquement cela qu'il pensait. Beaucoup de gens pensaient comme lui, ou à peu près comme lui et ils se considéraient comme des théosophes. Il ne voulait point être un des leurs... ${ }^{5}$.

6 En quête de perfection, Hugo prend cette même voie, mais se consacre surtout à l'Évangile. Seul le Christ était arrivé au but qu'il s'était fixé :

Jésus-Christ, le Christ seul, crucifié, avait atteint ce but élevé. Les autres cherchaient toujours ${ }^{6}$.

et plus loin :

Le Christ voulait que chacun trouve, rayonne, règne et triomphe; croire en lui : cela ne suffisait point ${ }^{7}$.

8 Les Évangiles nous fournissent des éléments de réponse, pour autant que l'on admette les interférences entre le monde de la religion, de la raison qui peut en affranchir l'individu et de l'imaginaire sans lequel la parole n'aurait pas de vie. Cette réponse est valable pour tous les personnages de Couperus en quête de perfection: ils ont une conception particulière de la personne idéale, représentée par le Christ ou par la Vierge Marie ${ }^{8}$. Dans ce contexte les prophéties de l'Ancien Testament et l'annonce de l'Apocalypse sont d'une importance évidente.

9 De Stille Kracht présente, comme les autres ouvrages de jeunesse et bon nombre d'œuvres ultérieures, cette quasi-fatalité multiforme qui pèse sur les protagonistes. C'est notamment le cas pour les œuvres qui se situent entre Eline Vere et Metamorfose, 
marquées d'une vision biblique et dans lesquelles les personnages ont une attitude mystique et citent la bible, alors que la narration elle-même est truffée d'images bibliques9. Il s'agit notamment du triptyque Noodlot (Fatalité) (1890), Extaze (Extase) (1891) et Eene Illuzie (Illusion) (1892), ouvrages que résume la très courte nouvelle Een Verlangen (Désir) (1890).

Dans Noodlot, Frank, le héros, est empêché d'aimer la femme qu'il s'est pourtant choisie, à cause de la présence de Bertie, aimable tir au flanc, oisif parasitaire. Extaze est le récit de Quarts, homme du monde aux nombreuses maîtresses, qui désire vénérer Cécile comme une madone, femme-vierge. Cécile, veuve d'un homme qui ne l'avait jamais aimée comme elle l'aurait souhaité, est prise d'une passion physique pour cet homme de mauvaise vie qui reste en même temps inaccessible pour elle parce qu'elle est ainsi faite. Eene Illuzie et Een Verlangen présentent le même cas de figure: dans Eene Illuzie, Tila, auteur de Jezus van Nazareth aspire à l'amour d'un homme qui, tout comme Quarts, ne peut que la vénérer comme une vierge. Carel Armand, l'ami de Tila, ne peut conjuguer la réalité d'une femme le désirant plus que tout au monde et ses propres illusions de bonheur dans l'amour platonique : tout simplement il ne la désire pas. Een Verlangen résume une fois encore le thème de l'auteur inconnu d'un récit autobiographique. Ces récits analogues forment un triptyque par leurs parallèles et inversions, qu'accompagne un quatrième récit dont le héros avoue être submergé par le sentiment. Anonyme, il n'est nullement romantique et analyse son problème avec beaucoup de clairvoyance, même s'il n'en relève pas le caractère profond. Tous ces protagonistes se trouvent sans exception dans l'impossibilité de vivre une véritable union amoureuse. Couperus utilise dans Extaze sa " plume mystique ", comme plus tard dans Metamorfose sa "plume théosophique " pour incarner dans ses personnages des idées. Quarts dit, par exemple, employer des paroles mystiques pour exprimer la vénération qu'il ressent pour Cécile, comparée à une madone. Il compare Christie, le fils de Cécile, au Christ - et ceci dans un contexte où les Essays de Ralph Waldo Emerson apparaissent comme le livre de chevet de Cécile. L'héroïne cherche, comme Tila, autre chose dans sa vie, une ascension spirituelle pour échapper à la routine quotidienne. Et Emerson distingue l'amour physique de l'amour spirituel qu'il veut indépendant de la beauté. Cécile s'inspire de cette source pour l'élévation de son âme, mais ne tarde pas, néanmoins, à être transportée par tous ses sens quand elle rencontre l'homme qui les compare, elle et son fils, à la madone et au Christ. Elle ressent le «Nirvana gris " comme les jeunes gens de son temps, et est soumise au sensitivisme, ce sentiment de revivre un passé lointain, autre phénomène littéraire fin-de-siècle qui prélude au symbolisme. Ce rappel est sans conséquence: comme les "plumes" différentes qu'utilise Couperus, c'est une allusion de plus au passé, ainsi mis en place par strates successives. C'est l'époque où Van Deyssel, auteur naturaliste des années 1880, publie dans la revue De Nieuwe Gids un article intitulé «La mort du naturalisme " ${ }^{11}$ et annonce l'importance du symbolisme qui cherche à percer le mystère divin. Couperus n'ignore pas ces courants littéraires, les mentionne même explicitement. Il élabore, pourtant, son propre style et utilise ces modes comme autant de paravents. Dans ce contexte, on n'échappe pas, d'ailleurs, à un parallèle entre Extaze et Un cour de femme de Bourget, à cette différence que le milieu socio-culturel de Quarts et de Cécile est protestant: le pardon y trouve difficilement sa place, comme le rappelle Quarts :

Je ne sais pourquoi, je me sens si... Peut-être est-ce à cause de votre pardon. La religion catholique est extraordinaire en ce qui concerne le pardon. Quelle consolation pour les hommes faibles ${ }^{12}$. 
11 L'idée sera reprise dans Van oude men sen, de dingen die voorbijgaan (Vieilles gens et choses qui passent) terminé en décembre 1904 : la fille de l'héroïne hantée par son passé parce qu'elle a un meurtre sur la conscience, se convertit au catholicisme et prie pour le salut de l'âme de sa mère. Cette idée de salut ainsi que l'image de l'homme faible devant un Dieu intransigeant renvoient à l'Ancien Testament et permettent de poser le problème de la morale chrétienne dans ces œuvres littéraires.

\section{La Bible, génératrice d'un héros incomplet}

12 C'est par la lecture ou l'évocation de la Bible que les personnages féminins prennent de l'épaisseur. Contrairement aux romans mystiques de l'époque, comme le Johannes Viator de van Eeden, l'écriture reste descriptive et extérieure, à part quelques versets de la Bible qui servent à focaliser les personnages.

Extaze illustre parfaitement les chevauchements entre réalité biblique et quotidienne d'une part, l'imaginaire de l'autre. L'auteur y utilise la Bible pour donner une tension dramatique à son récit, désacralisant, ainsi, le texte sacré. Avant l'arrivée de Quarts, le narrateur met en scène Cécile lisant la Bible à ses enfants. Elle leur lit le passage de la Genèse où Juda propose à son frère Joseph de rester auprès de lui à la place de Benjamin, leur frère cadet, pour éviter à leur père d'inutiles souffrances. Joseph, alors, révèle sa véritable identité (Genèse 45). Par rapport à l'Évangile, ce passage apprend la bonté de Juda envers Jacob, son père qui permettra la libération de Benjamin et surtout la réunion de la tribu dispersée, Joseph et Benjamin étant, contrairement aux autres frères, fils de la femme préférée de Jacob. Juda est ainsi campé comme l'homme fort et généreux, comparé à un lion (1 Chron. $5: 2$; Gen. 49 : 9,10; Apoc. $5: 5$ ), celui qui aura naturellement - dans sa descendance un roi - David - et même mieux, le Christ, comme le révèlent Esaïe ou encore Isaïe. Cette dernière référence n'est pas innocente, puisque c'est dans le livre du prophète Isaïe que le peuple est au centre et que Dieu annonce l'histoire de son peuple jusqu'à l'Apocalypse ${ }^{13}$.

Cette lecture terminée, Christie, le fils de Cécile, se montre déçu par le dénouement. Sa mère n'a pas le courage d'invoquer l'authenticité de l'histoire biblique. Elle met en doute, par contre, l'éducation qu'elle lui donne et s'attriste d'en imaginer les résultats, reflets de sa propre ignorance et de ses propres interrogations. Les contradictions de l'héroïne s'amplifient par les parallèles de situation - celle des enfants de Cécile et celle de la descendance de Juda - et induisent une réflexion sur l'importance de la morale.

Dans Eene Illuzie, Tila est également assaillie par des mouvements contradictoires. Après avoir écrit un livre sur la vie de Jésus, elle se rend compte, en se comparant à son héros, que pour elle la vie ne peut être faite de souffrances endurées pour les autres alors qu'elle se réduirait elle-même à être chaste :

Dieu, comment était-ce possible, comment! C'est elle qui avait été le créateur de tout cela, elle a eu cette vision supérieure, elle avait entendu en elle cette mélodie entrainante de la grande tragédie de la souffrance [...]; c'est elle, cette femme fragile, dont l'imagination avait accouché de cet être humain, lui le martyr de ses illusions chimériques de sacrifice, martyr qui croyait que ses sacrifices le rachèteraient! La vérité massacra l'idéal, l'embrocha sur sa lance, monta l'éponge de vinaigre vers les lèvres de l'idéal... ${ }^{14}$ temps Marie Madeleine, incapable d'effectuer l'ascension morale tant rêvée. Le rappel 
de l'Évangile renforce l'effort surhumain de Tila pour se sortir de ses désirs égoïstes. L'idéal de Jésus est pourtant subitement réfuté, car il ne correspond plus à l'état d'âme de l'héroïne. Jésus, dans sa dimension humaine - et non pas le Christ - sert d'élément de comparaison autant que d'enjeu dans la désacralisation.

Les protagonistes de Couperus visent à la perfection, tentent de s'y projeter comme les théosophes, mais l'image biblique, en l'occurrence celui de l'échec, subit un glissement de sens par la transformation que lui fait subir Tila, confrontée à la réalité. Au Jésus de l'Évangile se superpose l'homme victime de ses illusions chimériques, créé de toutes pièces par Tila, portant ainsi atteinte à l'historicité de Jésus de Nazareth ${ }^{15}$.

La quête d'un idéal à laquelle il est si souvent fait allusion trouve sa source dans Jean 19,29, Marc 15,36 et Mathieu 27,48 auxquels semble renvoyer la citation déjà reproduite d'Extaze. Elle se traduit dans une écriture qui est autant illusion qu'idéal déchiré. Ce que l'Écriture a annoncé est arrivé, mais cette "vérité massacrante » est bien amère pour celui qui, par définition, souffre : le héros romanesque tout comme le lecteur. C'est cette "vérité massacrante » la problématique du personnage coupérien. Comme Jésus dans La Vie de Jésus de Renan, le messie de Tila représente cet homme fin-de-siècle qui cueille chez Couperus les fruits amers de ses aspirations inaccessibles. Si le personnage de Tila semble avoir des prédispositions au "sensitivisme ", la conclusion détruit cette illusion, car à la fin de l'histoire, une fois que Carel aura dit son refus, elle se transforme en une femme tentatrice et hystérique. L'imaginaire l'emporte. Le lecteur assiste ainsi à la genèse d'un autre type d'héroïne et voit naître sous ses yeux une femme qui est fatale par la peur que sa déraison inspire. C'est elle qui sera contestée, et non pas la Bible. Le glissement est de taille, parce qu'il génère de nouvelles mythologies.

Dans ce triptyque, les problèmes psychologiques se manifestent partout, ramenés toujours à une condamnation implicite de l'union physique, thème en vogue partout en Europe dans ces années 1880 , avec Tolstoï prônant une vie saine, simple et chrétienne, comme dans Le Bonheur conjugal de 1864.

Ce thème est problématique pour Couperus lui-même. L'emploi des images bibliques tant condamné par les critiques néerlandais de l'époque ne sert point à défaire les images de leur sens. Au contraire. Il marque l'intensité avec laquelle se présente le problème personnel. L'interprétation de Tila est la conséquence de sa désillusion, son malheur et son amertume se projetant sur les souffrances du Christ. On s'étonne d'autant que les critiques de l'époque - dont J. van Loenen Martinet - aient pu dire que "plus rien ne rappelle la religion » ou encore que «tout est subjugué à la fatalité »" Les héros ont chacun leur place au sein d'une structure qui est celle de l'ego de Couperus où la trame biblique le dispute à une Écriture laïcisée par l'artiste. Ce qui s'appelle "noodlot » (fatalité) dans son œuvre est à la fois le destin et la conséquence voire la punition de ce à quoi ses héros aspirent au fond d'eux-mêmes, comme il y aspire au fond de lui-même. Ce héros luttera contre ces aspirations par le biais de la notion de pureté. Dans Een Verlangen, écrit à Paris en 1890, le narrateur note :

Dans son âme, il sentait un feu, sacré, d'amour inutile, qui ne brûlait pour aucun Dieu, là sur l'autel, isolé de son âme. Était-ce de sa faute s'il n'avait jamais trouvé ce dieu, s'il n'avait jamais rencontré un être, un seul, auquel il se consacrerait comme à lui-même ? [...] ou était-ce la fatalité qui crée des êtres, des êtres dotés de tous les talents du monde, et à qui il manque une seule chose que l'être humain désire avant tout, qui constituerait son bonheur, l'objectif même de son existence, la solution de son énigme vitale [...] était-ce le caprice de la Providence ou la Fatalité ou ? - pour 
lui offrir ce qui fait la joie de l'être humain et pour l'en priver : Ah, tant de choses :

ce qui était pour lui essentiel ${ }^{17}$.

21 Ce narrateur - autobiographique - cherche l'amour ou la passion comparée à une divinité. À travers un langage énigmatique, il évoque ce qui le préoccupe : sa vie sexuelle. La comparaison de l'amour à un dieu révèle combien ce divin reste inaccessible pour lui, à cause de son homosexualité, ce qui explique aussi pourquoi il va même jusqu'à évoquer le diable qui pourrait se cacher derrière l'interrogation: "caprice de la Providence» (une «Voorzienigheid» qui est synonyme de Dieu en néerlandais), la Fatalité (grecque) ou le diable, issu de sa propre culture chrétienne et plus particulièrement protestante. La théâtralisation qui sera la marque de l'univers coupérien et qui est portée notamment par le style est comme l'effet de cette tension intérieure. Elle approche au plus près ce qui le hante depuis des années autant qu'elle exprime sa propre souffrance : elle porte la marque de l'impossible perfection et de ce « presque » qui le fera toujours souffrir, un " presque » qui correspond à l'imperfection que le narrateur anonyme de Een Verlangen retrouve aussi bien dans l'art que dans la vie quotidienne. En discutant de cette perfection dans l'art avec Brauws, l'ami sculpteur, le héros répond :

Tu raisonnes et ressens comme un artiste et moi je n'en suis pas un ; je ne suis qu'un être dominé par ses sentiments et pour un tel être l'imperfection incarne la souffrance.

Et d'évoquer la Genèse pour la pureté des sentiments de l'homme avant sa chute et l'enfant qui représente cette pureté dans la théosophie. Le héros cherche le retour à la véritable pureté, comme le montre la description de son entourage :

Le monde était «presque » et pire encore, le monde était usé et souillé. Ah, comme son âme en souffrait, qui n'avait pas un seul idéal comme une divinité, mais qui aspirait tout, comme le Paradis! Le monde était vieux et [...] Ah, cette impureté, cette souillure et la lèpre du monde! Elles se trouvaient partout dans la boue des chemins, dans la sueur de son corps, dans la conscience animale de son âme! Toute cette lumière de l'origine de la vie, toute la pureté du début du monde, le paradis de la Genèse ${ }^{18}$.

La vie intérieure du narrateur et des autres personnages se présente sous forme d'images que les critiques de l'époque dénoncent, parce que pernicieuses. En réalité, les héros vont vivre une vie symbolique, même allégorique. Chaque personnage incarne une idée plus ou moins développée et ce sont ces idées que les critiques considèrent comme chimériques ${ }^{19}$. Les protagonistes sont toujours identiques et restent dans la plupart des cas énigmatiques dans leur quête d'une relation platonique. Leur analyse fait apparaître une fréquence des mêmes structures ouvertes et un fonctionnement manichéen : ils se font écho, comme le bien et le mal. Leur psychologie est ambiguë : Eline Vere, par exemple, mécontente de son sort, habillée de gris, sera mise en scène par l'auteur qui la présente en l'associant à une vieille femme, vue par la fenêtre et tout autant habillée de gris. Cette théâtralité dans le fonctionnement des héros a pour conséquence que le lecteur a tendance à les juger. À tout instant, le raisonnement du lecteur est mis en éveil et suscite le jugement, alors que le héros se débat dans l'opposition entre le présent et l'avenir, entre être et devenir.

Les idées et visions des personnages sont à double tranchant: Cécile est persuadée qu'elle a aimé son mari, prévenant quoiqu'un peu froid. Le portrait qui nous en est dressé comme un tableau de Rembrandt éclaire plutôt la froide distance et montre le contraire. Voilà pourquoi Cécile a soif d'amour et pourquoi, aussi, elle n'a pas 
véritablement envie de faire ce portrait. Le clair-obscur en question privilégie la psychologie et laisse le non-dit, tant présent dans l'œuvre, agir comme une force «fatale »; l'impossible union physique, thème constant, se distille au fur et à mesure que les récits avancent et nourrit la fatalité, comme dans Noodlot. Le mal s'annonce comme un malheur qui gronde au loin et déclenchera ensuite le déluge. Le roman place le lecteur en pleine fable biblique où chacun a sa place à l'avance. Le malheur est là, dès le départ. Dès lors les jeux sont faits. Cette structuration n'est pas affaire de simple fatalité. Au contraire, c'est la constellation, le récit épique ou l'interprétation même de l'existence des héros qui varient peu. Comme dans la Bible, le bien et le mal y coexistent, tout comme dans la vie quotidienne. Les références se font tantôt à l'Évangile avec le Christ comme image de la souffrance, tantôt à l'Ancien Testament et à l'Apocalypse de Jean avec l'annonce d'une fatalité chrétienne sous forme de jugement dernier. La fatalité se manifeste sans exception chez ces personnages placés devant des choix impossibles. Dans Extaze elle envahit Cécile, femme respectable et qui le reste, contrairement à Quarts, incarnation du mal, celui dont elle est " éprise ", comme sortie d'elle-même. Dans Noodlot, c'est Bertie qui incarne ce mal, mais c'est encore la femme pure qui sera le plus aux prises avec la fatalité et qui en ressentira la force et les effets. La vraie raison de la chasteté, tant prônée et réclamée, n'est donc pas morale. Bertie figure le mal par son oisiveté et sa volonté d'entraver l'amour de Frank et d'Eve, entrave nécessaire à la sauvegarde de sa propre existence. C'est l'homme androgyne dans un décor oriental, tout comme Vincent Vere. Ce même motif apparaît comme un fil conducteur dans Metamorfose, où Hélène, ayant vécu dans le péché, se refuse à accepter l'amour de Hugo, s'en considérant indigne. Les noms sont hautement symboliques et contribuent à densifier les tensions du récit et à renforcer le poids mythique: Eve, Cécile, vierge et martyre du cinquième siècle, fiancée à Valerien, le païen qui se voit contraint de respecter sa virginité, Tila - qui pourrait être un Thilo déguisé, auteur d'un ouvrage sur le christianisme du temps de Couperus - Hélène, Quarts, qui signifie cristal de roche avec ses variétés colorées, améthyste, jaspe, œil-dechat, agate ou calcédoine. Tous ces éléments sont évocateurs - pour un lecteur familier de la Bible - de la ville sainte de Jérusalem telle qu'elle apparaît dans l'Apocalypse. D'autres noms, par contre, Frank, Carel voire Eline s'inscrivent dans d'autres réalités, comme Bertie qu'Eve considère comme une femme, tant son comportement est efféminé :

Et son âme romantique (d'Eve) attribua la douleur de ce sourire à l'apparition poétique d'une jeune divinité ou d'un ange déchu: la douceur d'un être mythologique asexué... ${ }^{20}$.

Bertie est un personnage intéressant dans la mesure où, incarnant le péché, il désire Frank non pas en tant qu'objet de désir, mais pour le garder pour lui tout seul. Il entraînera, comme Vincent, sa victime vers sa perte, encore qu'on ne puisse pas dire que Frank reste passif. Contrairement à Eline, il prend son destin en mains, élimine son mauvais génie, mais c'est au prix de sa propre existence, dorénavant vouée à la déchéance. La fatalité qui s'opère dans Noodlot n'est pas celle qui frappe Eline Vere: celle-ci succombe à la tentation du suicide, alors que Frank devient meurtrier. L'univers des protagonistes est comme le «logos » de la Bible, parole écrite de ce qui devait être. $\mathrm{Au}$ sein de ces ouvrages, le Christ détermine le déroulement: la chute d'Adam est annoncée comme celle de Belchatsar, la mort de Jésus et l'Apocalypse. Le rapprochement avec l'Ancien Testament et l'Apocalypse n'est guère étonnant, quand on voit, dans Metamorfose Hugo comparé à ce roi Belchatsar, passé à la postérité 
culturelle parce qu'incapable de lire le «Mene tekel »- thème qui a inspiré peintres et poètes récemment encore. Mais Hugo s'échappe de ce mal par son mariage avec sa cousine, ce qu'il appelle son « petit bonheur ».

\section{Symbolique des personnages} plane sur les héros autant que l'omniscience de l'artiste-créateur, dépositaire du savoir et tout puissant. La figure de Jésus, par contre, intervient pour traduire la problématique du héros, ce qui fait qu'il devient figure emblématique, par delà l'exégèse traditionnelle. Ce glissement de sens place le travail littéraire dans une filiation rationaliste dont il est la conséquence directe.

1878, Allard Pierson avait nié l'historicité du Christ dans son Bergrede (Sermon sur la montagne) et dès 1856 le docteur de Lespinasse avait établi une comparaison entre les peuples orientaux et chrétiens. En 1884, A.D. Loman avait fait paraître dans De Gids un article sur l'origine du christianisme qu'il ne situait plus au premier, mais au deuxième siècle, contestant implicitement les lettres de Paul. Ce débat qui n'a rien perdu de son actualité place l'exégèse dans une perspective symbolique où, après Jésus de Nazareth, le Christ est un nouveau Dieu chrétien, même un être mythique. Couperus a-t-il suivi cette interprétation humaniste de la Bible? Le passage déjà cité, où Juda veut se sacrifier pour son père se poursuit avec l'annonce faite par Benjamin que le descendant de la tribu de Juda sera un futur roi, un lion qui, seul, aura le droit de révéler le message apocalyptique, le Christ en l'occurrence ${ }^{21}$. C'est la source par laquelle s'explique le souci que les personnages coupériens ont de leur avenir. Les récits de jeunesse montrent l'omniprésence d'une angoisse à propos d'un avenir incertain. Le passage de Juda, déjà relevé, aussi bien que l'histoire de Jésus conçue et interprétée par Tila placent au centre des problèmes le sacrifice du fils par le père, alors que, ailleurs, on trouve des allusions à peine déguisées au père castrateur, notamment dans Een Verlangen et Metamorfose. Et partout, la souffrance des personnages est comparée à celle de Jésus, présenté comme le frère de l'homme s'occupant des faibles. Le péché de Quarts est défini par Cécile comme "Avec moi, il sent son côté humain et non pas cette autre chose ", description assez vague, mais qui prend de l'épaisseur par le rappel de la "bête", motif très présent dans la littérature néerlandaise, rappel de la bête de l'apocalypse que tout homme porterait en soi. Cécile est assaillie par la vision suivante :

Quelque chose l'envahit comme le noir des nuages et elle eut peur de ce qu'elle ressentait en elle-même, comme un courant indéfinissable tout noir, comme si le rivage était couvert de boue, comme si cette boue montait en cercles troubles, grandissant toujours! Elle eut peur de cette inondation et ne voulut point la voir, mais le noir se répandait dans les paysages - autrefois illuminés des rayons de l'horizon - sous un ciel d'encre dessiné comme la nuit éternelle ${ }^{22}$.

Vision apocalyptique d'un déluge noir en rapport avec l'affect du personnage, comme elle hante Frank, sur le point de commettre son meurtre :

Il vit tout en rouge : pourpre et rouge écarlate et vermillon, des roues ensanglantées qui tournaient autour de lui [...] cela tournait toujours autour de Frank, comme des faiblesses pourpres, des idioties vermillon, des cauchemars rouge sang $^{23}$. 
Dehors, à travers les vitres embuées, elle vit le ciel tragique chargé de nuages qui courent et obscurcissent le ciel et elle vit la pluie tombant avec fracas comme le déluge, elle vit la mer à travers ce voile de pluie torrentielle, sombre, menaçante comme un danger ${ }^{24}$.

La pluie se transforme pour Eve en vision diluvienne, rappelant celle qu'elle avait eue déjà avant le drame et qui devient, de ce fait, prémonitoire. L'auteur recourt dans la description qu'il en fait à un langage simple, phrophétique, avec abondance, cependant, de conjonctions de coordination et de répétition de pronoms en style indirecte libre. La phrase épouse le style biblique, avec un déplacement des compléments pour augmenter la charge théâtrale.

Après 1897, la fréquence et l'importance des visions bibliques s'amoindrissent considérablement. On constate que jusque là la fréquence des visions évangéliques et apocalyptiques conforte finalement les personnages. Ils sont tourmentés, certes, mais leur pendant négatif seul est comparé à des êtres mythologiques, Quarts à Nimrod, le Centaure aux yeux de Jules ou encore Vincent et Bertie, êtres androgynes évoluant dans un décor mythique oriental. Ils sont aussi indispensables, dans l'incarnation du mal, que les héros véhiculant des valeurs bibliques. Ils fonctionnent dans le récit comme moteur d'une exégèse. Bertie représente l'obstacle, voire le mal, sert d'antagoniste pour "camper» le problème à la manière théâtrale. Lorsqu'il est question de traduire Noodlot en anglais en 1891, Clara Bell, la traductrice propose de composer le titre à l'aide du nom de "viper ", vipère, pour qualifier Bertie ${ }^{25}$. Couperus refuse la proposition et tient à maintenir le mot de fatalité. Or, pour le public chrétien et très puritain de l'époque, dans une société où l'église détermine toujours les mœurs, Bertie incarne le mal. Mais cet antagoniste fort peu sympathique sert à voiler comme à dévoiler le problème. Le titre trop négatif à l'encontre de ce personnage contesté ne peut qu'être douloureux pour celui qui l'a crée comme une part de lui-même. Il en va de même pour Quarts dont le nom évoque pour Cécile la cruauté des sons, mais dont le signifiant "pierre précieuse » maintient dans l'esprit de tous une connotation positive. Dans ces différents cas, il s'agit de renversements des valeurs symboliques. Vincent et St. Clare, Jules et Quarts, Bertie et Frank sont des couples-miroirs. L'amour que ressent le jeune Jules pour Quarts pourrait apparaître anodin, aux yeux du lecteur, comme celui que le petit Karel, dans Een Zieltje (Une âme), conçoit pour son oncle. Mais une fois adolescent, Karel l'admire toujours, ce qui sera l'origine du rejet qui le frappe et explique son suicide.

\section{Évolution vers la mythologie}

Le cheminement depuis Eline Vere jusqu'à Metamorfose est de taille : le héros va opter pour la vie, en y laissant une part de lui-même, comme ce fut le cas aussi bien pour Jésus que pour Couperus. À la fin de Metamorfose, il s'avère incapable de se consacrer au monde citadin et à la misère, selon l'exemple que donnent les textes sacrés. Le héros va se consacrer à Emilie, sa future femme, en réalité Elisabeth Baud, cousine de l'auteur. Etrange mélange de réalité et de fiction, d'écriture romanesque et d'une finalité littéraire qui s'affranchit du poids de la Bible pour s'ouvrir à la vie :

Et pour la première fois, il apprécia... Les lettres sur les murs immenses de son existence « Même, mene... » pâlirent. 


\section{s'estompe, s'éloigne. Le héros n'est plus menacé, parce qu'il accepte enfin sa condition} humaine. Une période mouvementée de la vie de Coupérus prend fin, dans laquelle les éléments bibliques ont été le moteur d'une évolution personnelle et d'une écriture littéraire dans laquelle le mythe a constitué comme un contrepoids. Beaucoup de questions restent en suspens, notamment par rapport à la place de la femme dans cet univers imaginaire. Car, en effet, force est de constater que tous ces personnages évoluent dans un monde observé par la femme. À plusieurs reprises cette femme est décrite physiquement comme celle du Cantique des cantiques alors qu'à d'autres endroits elle prend la forme allégorique qui justifie une comparaison avec ce que représentait l'Église pour le Christ, selon les chrétiens notamment les catholiques ; Eve, Cécile, Tila, trois femmes, toutes trois incarnation de la pureté, trois azalées, rouge, noire et blanche. Elles sont d'une pureté factice : la femme rouge est faite pour aimer, la noire porte encore le deuil, mais est prête à vivre un nouvel amour, la troisième n'est blanche qu'en apparence puisqu'elle aussi cherche l'amour charnel - au point d'en devenir hystérique. Et pour parfaire le tableau, rappelons qu'en grec « azalée » porte le signifiant « desséché ». Ces héroïnes sont en même temps hiératisées et reconnues dans leur action indispensable. Elles font écran autour des héros, Frank, Quarts et Carel dont elles motivent l'évolution dramatique positive: Frank se trouve, certes, dans l'impossibilité de se détacher de Bertie et finira par le tuer. Quarts aura envers Jules une attitude moins ambiguë voire paternelle, encore que suspecte aux yeux de Cécile. Il reste ambigu comme création, à la fois objet d'amour de la part de Jules et homme à femmes. Rappelons que c'est la période qui représente, dans la vie de Couperus, une rupture avec le passé, puisqu'il épouse sa cousine en 1891, après le départ de son ami Ram. F. Bastet rappelle utilement, à ce propos, que la figure de Tila pourrait s'inspirer de Minta, autre cousine qui aurait voulu l'épouser.

Ces trois femmes dans leur interdépendance et leur signification symbolique figurent les différentes étapes d'une vie, le meurtre de Bertie servant à représenter la rupture de deux êtres. L'auteur évoque, en 1905, cette période comme celle des grandes souffrances. Dans l'évolution créatrice, tellement imbriquée dans ces éléments biographiques, le «troisième larron » après Frank et Quarts apparaît bien plus serein : par opposition à Tila à la fois parfaite et tentatrice, Carel - dépourvu d'antagoniste, rappelons-le - sera serein, mais au prix d'une solitude assumée.

Quand on cherche à pénétrer le mystère de ces personnages et à définir leur fonction dans un univers chavirant entre le devoir moral et un imaginaire qui est autant le lieu d'une confession qu'il représente une échappatoire, Metamorfose semble apporter un début de réponse : Hélène y porte bien son nom puisque c'est la femme parfaite, ayant vécu dans le péché. L'union amoureuse ne se réalise pas. À travers elle - référence grecque - Jésus, le compatissant, sera transformé en Christ.

Les œuvres qui suivent en disent long sur ce que signifie cette sérénité retrouvée, acceptation de soi dans l'harmonie de l'autre. Psyche (1898) et Fidessa (1899) sont des contes symbolistes qui servent en quelque sorte d'épilogue et résument le choix que Hugo et Couperus lui-même ont fait du mariage, ce "petit bonheur" dont parlait Metamorfose. Psyché et Eros, dans le royaume d'avenir, et Fidessa et Sans Joye, chevalier accompagne de la licorne symbole de chasteté persan autant que médiéval, renvoient à l'union de deux êtres. Mais là où Extaze, par exemple portait, comme ce fut le cas dans la tragédie hollandaise du «Siècle d'Or » un sous-titre explicatif, Récit du bonheur et de la 
douleur en l'occurrence, Psyche et Fidessa se présentent d'emblée comme des contes. C'est que l'auteur a dépassé le stade de l'interrogation personnelle et que, plongé dans l'univers littéraire et notamment mythologique et classique, il «portera la parole »une parole affranchie. L'analyse de la traduction qu'il fera de la Tentation de Saint Antoine de Flaubert pourrait montrer combien il utilise, dorénavant, les sources bibliques et post-bibliques dans une perspective esthétique et littéraire.

\section{NOTES}

1. - Cf. G. Van de Louw, «"Holländisch” une "Flämisch” im Auge Prager Bürger (1870-1939) » in Der Niederländische Sprachraum und Mitteleuropa, Leopold Decloedt/Herbert van Uffelen (Hg.), Böhlau Verlag Wien, 1995, pp. 58-64.

2. - Bulkboek 1992.

3. - Ces récits, nés dans un contexte naturaliste, s'inscrivent dans la littérature fin-de-siècle en Europe. Leur auteur s'est toujours prévalu d'être un dandy. Dans Metamorfose, il se décrit parfois comme un véritable dilettante et se vante de s'être servi de "toutes les plumes " pour arriver à ses fins. Ces «plumes » qui reflètent la mode de l'époque ne sont qu'un moyen pour arriver à ses fins et constituent l'aspect le moins intéressant de l'œuvre, car elles servent d'écorce pour masquer quelque chose de plus profond. Elles sont un moyen assez typique pour l'auteur qui entend ainsi cacher son jeu.

4. - Treize ans plus tôt avait été fondé à New York par le colonel Olcott, son président, et H.P. Blavatsky l'Association Théosophique qui visait à instaurer une nouvelle fraternité entre les hommes. Madame H.P. Blavatsky avait écrit en 1889 The key to theosophy, dans lequel elle fait mention de la philosophie de R.M. Emerson. Pendant les années 1880, celui-ci fait publier ses œuvres complètes, intitulées The complète Prose Works of Ralph Waldo Emerson avec des Essays, dans lesquelles il évoque entre autres l'incompatibilité entre l'esprit et l'amour physique. Aux PaysBas, le représentant le plus important des théosophes est madame Annie Besant qui, à partir de mai 1892, écrit régulièrement dans Theosophia, revue théosophique fondée la même année.

5. - Verzamelde Werken, Amsterdam, G. van Oorschot, III, p. 234.

6. - Idem, p. 236.

7. - Idem, p. 237. C'est nous qui soulignons.

8. - Le sens du titre Metamorfose s'explique par la mise en abîme révélatrice du livre dans le livre : l'homme non seulement est croyant, mais est à la recherche du «bien-faire » comme le Christ. La métamorphose est d'une grande importance dans la théosophie où l'homme cherche à accéder progressivement à un niveau supérieur de pureté.

9. - Les « romans des rois » n'entrent pas en compte ici pour la simple raison que leur figuration biblique très restreinte ne correspond pas à une fonction déterminante au sein de l'œuvre et n'approfondit pas davantage la problématique du héros.

10. - Extaze, Volledige Werken, L.-J. Veen, 5, p. 12.

11. - De Nieuwe Gids, 1890-1, deel 2.

12. - Extaze, Volledige Werken, 5, p. 73.

13. - Es. $53: 1-11$.

14. - Eene Illuzie, Volledige Werken, 6, p. 43. 
15. - Dans Leven in extaze, Amsterdam, Querido, 1983, Jan Fontijn suppose que les personnages de Couperuis sont divinisés comme les personnages mystiques de la littérature néerlandaise. Ce serait vrai, si le texte était composé sur un modèle grandiloquent, attribuant aux personnages toutes les qualités telles qu'elles figurent dans la Bible. Ici ce n'est point le cas, étant donné que l'image du Christ donnée par Jean a été transformée, si bien que les patroles « lui le martyr de ses illusions chimériques de sacrifice" seraient plutôt l'expression de la désillusion totale. Tila va jusqu'à se comparer au Christ et se rend compte par la suite qu'elle a manqué de réalisme. Le Christ qu'elle décrit ressemble plutôt à celui de Renan.

16. - J. van Loenen Martinet, Het fatalisme in onze jongste letterkiinde, Haarlem, 1891, p. 29 cité par M. Galle, Couperus in de kritiek, A'dam, Polak et van Lennep, 1963, p. 14.

17. - Een Verlangen, Volledige Werken, 6, p. 61.

18. - Ibid., p. 66.

19. - Cf. Van Nés, Prozastukken 1897, p. 267.

20. - Noodlot, p. 53.

21. - Cf. Esaïe, 11, 53.

22. - Extaze, Volledige Werken, 5, p. 62.

23. - Ibid.

24. - Ibid.

25. - Cf. F. Baster, Louis Couperus, Een biografie, Amsterdam, Querido, 1987, p. 724.

\section{RÉSUMÉS}

Les premiers ouvrages de Couperus comportent notamment une série de trois livres, Noodlot, Extaze et Eene Illuzie qui forment un ensemble et trouvent leur écho dans Metamorfose, son roman autobiographique. De la structure de ces ouvrages très semblables ressort le thème de la chasteté. La fatalité, thème favori de Couperus, contenue dans les focalisations internes des personnages, est à la fois le destin, la providence et le diable auquel il fait allusion sans le citer. Elle présage l'arrivée de Jésus avec les Évangiles et l'Apocalypse. Le triptyque représentant l'évolution du héros masculin et construit en plus grande partie à partir du point de vue féminin, présente des personnages pleins de contradictions. Il en résulte une image esthétique trompeuse qui dissimule le problème du personnage masculin. En réalité ce triptyque représente l'évolution de l'auteur. Mais à la fin de celui-là, la symbolique plus claire pour le personnage masculin va de pair avec un glissement de valeurs pour le personnage féminin, qui répond de moins en moins aux valeurs chrétiennes et annonce la naissance de l'image mythique. Le renversement psychologique des personnages va embrouiller la symbolique et crée un système indépendant par rapport à l'exégèse biblique où le nouveau personnage coupérien trouve sa place. De même la dichotomie occident/orient va jouer un rôle de plus en plus important dans l'œuvre ultérieure avec la traduction par Couperus de La Tentation de saint Antoine de Gustave Flaubert.

Het jeugdwerk van Louis Couperus bevat een serie van drie boeken Noodlot, Extaze en Eene Illuzie die een geheel vormen en hun weerklank vinden in Metamorfose, een autobiografische roman. Een steeds terugkerend thema in de structuur van de sterk op elkaar lijkende werken is het kuisheidsmotief. Het noodlot, sterk aanwezig in de interne focalisaties van de personages, is het noodlot naar de opvatting van de Grieken, de voorzienigheid en de duivel, die niet expliciet genoemd wordt. Het noodlot kondigt de Evangelien en het laatste oordeel aan. De triptiek 
verhaalt vooral de ontwikkeling van het mannelijke personage maar is vanuit het standpunt van het vrouwelijke personage opgebouwd. De personages zijn echter vaak tegenstrijdig. Dit heeft een verkeerd esthetisch beeld tot gevolg dat de werkelijke problematiek van de man overschaduwt: de triptiek stelt in werkelijkheid de problematiek van de auteur voor. Hoe duidelijker de symboliek van het mannelijke personnage, hoe incoherenter de vrouw, die steeds minder aan christelijke normen en waarden beantwoordt en het mythische element aankondigt en de fatale vrouw. De psychologische ommekeer verdoezelt de symboliek en creeert een onafhankelijk systeem waar het nieuwe personage een plaats heeft buiten de bijbelverklaring. Ook wordt langzamerhand de dichotomie westers / oosters zichtbaar. Die openbaart zieh een paar jaar later met Couperus' vertaling van La tentation de saint Antoine van Gustave Flaubert.

\section{AUTEUR}

\section{MARJAN KRAFFT-GROOT}

Université Charles-de-Gaulle - Lille III 